\title{
The distance to the Pleiades from the eclipsing binary HD 23642
}

\author{
J. Southworth, P. F. L. Maxted and B. Smalley \\ Department of Physics and Chemistry, Keele University, Staffordshire, ST5 5BG, UK \\ email: jkt@astro.keele.ac.uk, pflm@astro.keele.ac.uk, bs@astro.keele.ac.uk
}

\begin{abstract}
The distance to the Pleiades open cluster is of fundamental importance to many aspects of stellar astrophysics but is currently controversial. The 'long' distance scale of $132 \pm 3 \mathrm{pc}$ is supported by main sequence fitting analyses, ground-based parallax observations and analysis of the astrometric binary Atlas. The 'short' distance scale of $120 \pm 3 \mathrm{pc}$ comes from parallaxes observed by the Hipparcos satellite. Munari et al. (2004) studied the detached eclipsing binary HD 23642 and found a distance of $132 \pm 2 \mathrm{pc}$. We reanalyse the data of Munari et al. to explore the different methods of estimating the distance of an eclipsing binary system. We use the surface brightness relations of Kervella et al. (2004) to find a distance of $139 \pm 4 \mathrm{pc}$, which is consistent with the 'long' Pleiades distance and in disagreement with the Hipparcos parallax distances to the Pleiades and to HD 23642 itself. By comparing the observed masses and radii of the binary with theoretical predictions we derive a metal abundance approximately equal to or slightly greater than solar. Further photometric observations of the binary are needed to improve the analysis.
\end{abstract}

\section{Introduction}

The Pleiades is a young open cluster situated in the solar neighbourhood. Due to its proximity the cluster has been exhaustively studied and its fundamental parameters, and distance, are considered to be well known. The 'long' distance scale of the Pleiades comes originally from Johnson (1957), who derived a distance of 129 pc from $U B V$ photometry. Crawford \& Perry (1976) found a distance of $128 \mathrm{pc}$ from photoelectric uvby $\beta$ photometry of 83 Pleiades stars. Vandenberg \& Bridges (1984) and Meynet, Mermilliod \& Maeder (1993) found a distance of $132 \mathrm{pc}$ by fitting model isochrones to the morphology of the Pleiades colour-magnitude diagram. An alternative method was adopted by O'Dell, Hendry \& Collier Cameron (1994), who used the Barnes-Evans relation to find the angular diameters of Pleiades members. This technique is subject to different systematic errors, but these authors also found a distance of $132 \mathrm{pc}$.

However, observations by the Hipparcos satellite (Perryman et al. 1997; van Leeuwen, these proceedings) give a 'short' distance of $120 \pm 3$ pc, based on the entirely geometrical method of trigometric parallax. This is in significant disagreement not only with previous estimates of the Pleiades distance but also with the distance of $131 \pm 7 \mathrm{pc}$ given by Gatewood et al. (2000) from ground-based parallax measurements. van Leeuwen (1999) has confirmed the distance based on the Hipparcos observations, and found that five other young open clusters have main sequences as dim as the Pleiades if the Hipparcos distance estimates are accurate.

Pinsonneault et al. (1998) and Soderblom et al. (1998) investigated this discrepancy in distance determinations by performing a main sequence fitting analysis of the Pleiades and by attempting to discover nearby subluminous stars with solar metal abundance. They concluded that the Hipparcos measurements were most likely wrong and also noted 
that the most discrepant measurements belong to stars in the centre of the Pleiades, where the stellar density is the highest.

Grenon (1999) has suggested that the Pleiades distance problem could be resolved if the cluster was metal-poor, and has measured an iron abundance of $[\mathrm{Fe} / \mathrm{H}]=-0.11$ dex from Geneva photometry. This is in conflict with the solar iron abundance found by Boesgaard \& Friel (1990) from high-resolution spectra of twelve F dwarfs in the Pleiades. Stello \& Nissen (2001) used uvby $\beta$ photometry to determine the distance to the Pleiades by shifting its main sequence to coincide with the colours and brightnesses of nearby stars with a similar metal-sensitive Strömgren index, $m_{1}$. The resulting distance, $132 \pm 2 \mathrm{pc}$, is in agreement with the 'long' distance scale and is not subject to metallicity effects. However, Castellani et al. (2002) were able to find a good agreement between the Pleiades main sequence, using Hipparcos parallax distances, and theoretical predictions from the FRANEC stellar evolution code, by using a subsolar metal abundance of $Z=0.012$.

Makarov (2002) pointed out that the method of calculating the parallaxes of stars from Hipparcos observations may cause small systematic errors in regions of high stellar density. He reanalysed the Hipparcos Intermediate Astrometric Data for the Pleiades, including in the analysis some numerical terms which were neglected in previous analyses (Perryman et al. 1997; Robichon et al. 1999). The resulting distance of $129 \pm 3 \mathrm{pc}$ agrees well with the 'long' Pleiades distance scale and casts doubt on the original Hipparcos results.

Recent interferometric observations (Pan, Shao \& Kulkarni 2004) of the astrometric binary Atlas (HD 23850) with the Palomar Testbed Interferometer give a distance in the range of 133 to $137 \mathrm{pc}$, supporting the 'long' Pleiades distance. Their results are also in good agreement with lunar occultation observations of Atlas. Munari et al. (2004, hereafter M04) measured the distance to the detached eclipsing binary HD 23642, a member of the Pleiades and found a distance of $131.9 \pm 2.1 \mathrm{pc}$, in agreement with the 'long' distance scale.

\subsection{The eclipsing binary HD 23642 in the Pleiades}

Detached eclipsing binaries (dEBs) with double-lined spectra are one of the best sources of fundamental astrophysical data (Andersen 1991) as their absolute masses and radii can be measured to accuracies better than $1 \%$. Such data can be used to provide a strict test of different stellar evolutionary models, and the distances to dEBs can be determined empirically to an accuracy of about $3 \%$. dEBs in open clusters are particularly useful because the age and chemical composition of the cluster may be combined with accurate values of the masses and radii of the $\mathrm{dEB}$ to provide an even more exacting test of theoretical models. Alternatively, the astrophysical parameters of dEBs in open clusters can be compared with theoretical evolutionary models to determine the chemical composition of the cluster. For example, Southworth, Maxted \& Smalley (2004a) studied the early-type dEBs V615 Per and V618 Per, in the open cluster h Persei, and determined a metal abundance of $Z \approx 0.01$. Southworth, Maxted \& Smalley (2004b) analysed the high-mass dEB V453 Cyg, a member of the open cluster NGC 6871, in order to test theoretical predictions of the masses, radii, effective temperatures and central condensation of early-B stars.

HD 23642 (Table 1) was discovered to be a double-lined spectroscopic binary by Pearce (1957) and Abt (1958). Abt \& Levato (1978) provided a spectral classification of A0 Vp (Si) + Am, where the metallic-line character of the secondary star relies on the presence of strong Fe I lines. Torres (2003) discovered shallow secondary eclipses in the Hipparcos photometric data of HD 23642 and also presented an accurate spectroscopic orbit. 
Table 1. Identifications and astrophysical data for the HD 23642 system.

\begin{tabular}{llc}
\hline & & Reference \\
\hline Henry Draper number & HD 23642 & 1 \\
Hipparcos number & HIP 17704 & 2 \\
Bonner Durchmusterung & $\mathrm{BD}+23^{\circ} 430$ & 3 \\
Hz number & $\mathrm{Hz} 540$ & 4 \\
$\alpha_{2000}$ & 034729.5 & 2 \\
$\delta_{2000}$ & +241718 & 2 \\
Hipparcos distance (pc) & $111 \pm 12$ & 2 \\
Spectral type & $\mathrm{A} 0 \mathrm{Vp}(\mathrm{Si})+\mathrm{Am}$ & 5 \\
$B$ & $6.898 \pm 0.015$ & 8 \\
$V$ & $6.819 \pm 0.015$ & 8 \\
$J$ & $6.673 \pm 0.030$ & 6 \\
$H$ & $6.625 \pm 0.030$ & 6 \\
$K$ & $6.631 \pm 0.030$ & 7 \\
Orbital period (days) & $2.46113400(34)$ & 7 \\
Reference time (HJD) & $52903.5981(13)$ & \\
\hline
\end{tabular}

References: (1) Cannon \& Pickering (1918); (2) Perryman et al. (1997); (3) Argelander (1903); (4) Hertzsprung (1947); (5) Abt \& Levato (1978); (6) 2MASS photometry transformed to the SAAO JHK system (see Moro \& Munari, 2000); (7) M04; (8) Tycho data from Perryman et al. (1997), transformed to the Johnson system using the results of Bessell (2000).

M04 derived precise absolute masses and radii of both components from five highresolution échelle spectra, and complete $B V$ photoelectric light curves. From consideration of the effective temperatures $\left(T_{\text {eff }}\right)$ and bolometric magnitudes of the components, and using the Wilson-Devinney light curve fitting code (Wilson \& Devinney 1971; Wilson 1993) they found the distance to HD 23642 to be $131.9 \pm 2.1 \mathrm{pc}$, where the quoted error is the formal error of the fit. We have reanalysed their data (which they have graciously provided on the internet) to investigate the advantages and disadvantages of different methods of estimating the distance of eclipsing systems.

\section{Spectroscopic analysis}

Table 2. Radial velocities, weights and $O-C$ values (in $\mathrm{km} \mathrm{s}^{-1}$ ) for HD 23642, taken from M04. For the SBOP analysis, observations were assigned weights of 2,1 or 0.5 if their quoted uncertainty was $0.5,1.0$, and $1.5 \mathrm{~km} \mathrm{~s}^{-1}$ respectively.

\begin{tabular}{ccrrr}
\hline HJD -2400000 & Primary velocity & $O-C$ & Secondary velocity & $O-C$ \\
\hline 53039.41273 & $-85.0 \pm 0.5$ & -0.4 & $134.5 \pm 0.5$ & 0.2 \\
53039.45356 & $-88.0 \pm 0.5$ & 0.2 & $139.0 \pm 0.5$ & -0.5 \\
53039.50548 & $-91.0 \pm 0.5$ & 0.4 & $143.0 \pm 1.0$ & -1.0 \\
53043.25049 & $105.5 \pm 1.5$ & 0.4 & $-136.0 \pm 1.0$ & -2.0 \\
53046.25318 & $29.5 \pm 0.5$ & 0.7 & $-24.5 \pm 1.0$ & 1.6 \\
\hline
\end{tabular}

M04 observed HD 23642 five times with the Élodie échelle spectrograph on the 1.93$\mathrm{m}$ telescope of the Observatoire de Haute-Provence. The derived radial velocities were combined with the spectroscopic observations of Pearce (1957) and Abt (1958), using lower weights for the older data, to calculate a circular spectroscopic orbit.

The low weight - and low precision - of the data of Pearce (1957) and Abt (1958) mean that they contribute little to the accuracy of the spectroscopic orbit. For comparison with the results of M04 we have chosen to derive the orbit using only the five échelle 
Table 3. Parameters of the spectroscopic orbit derived for HD 23642.

\begin{tabular}{llcc}
\hline & & Primary & Secondary \\
\hline Velocity semi-amplitude $\left(\mathrm{km} \mathrm{s}^{-1}\right)$ & $K$ & $99.10 \pm 0.58$ & $140.20 \pm 0.57$ \\
Systemic velocity $\left(\mathrm{km} \mathrm{s}^{-1}\right)$ & $V_{\gamma}$ & $6.07 \pm 0.39$ \\
Mass ratio & $q$ & $0.7068 \pm 0.0052$ \\
Projected separation $\left(\mathrm{R}_{\odot}\right)$ & $a \sin i$ & $11.636 \pm 0.041$ \\
Minimum mass $\left(\mathrm{M}_{\odot}\right)$ & $M \sin ^{3} i$ & $2.047 \pm 0.016$ & $1.447 \pm 0.013$ \\
\hline
\end{tabular}

velocities for each star. The orbit was computed using SBOP $\dagger$, with the orbital ephemeris from M04, eccentricity fixed at zero, and equal systemic velocities for both stars. The radial velocities, adopted weights and observed minus calculated $(O-C)$ values are given in Table 2, and the parameters of the spectroscopic orbit are given in Table 3 . These parameters, plotted in Fig. 1, are in acceptable agreement with those of Torres (2003) and Abt (1958), although a little different to the values of Pearce (1957) (but see Griffin, 1995).

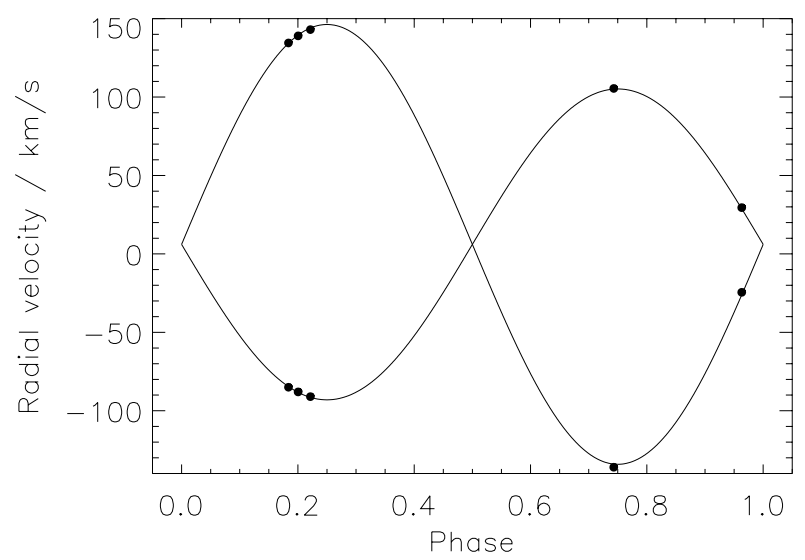

Figure 1. Circular spectroscopic orbit for HD 23642 derived using the Élodie radial velocities.

From comparison between the observed spectra of M04 and synthetic spectra calculated using UCLSYN and ATLAS9 model atmospheres (see Southworth et al. 2004a for references), the effective temperatures of the two stars were found to be $9750 \pm 250 \mathrm{~K}$ and $7600 \pm 400 \mathrm{~K}$, in good agreement with the results of M04. The uncertainties in these values include possible systematic errors caused by the slight spectral peculiarity of both components of HD 23642. The rotational velocities of both stars were confirmed to be synchronous with the orbital motion, in agreement with Zahn's (1977) theory of tidal evolution which predicts a synchronisation timescale of $0.5 \mathrm{Myr}$.

\section{Photometric analysis}

The $B$ and $V$ light curves contain 492 and 432 individual measurements, respectively, obtained with a $28 \mathrm{~cm}$ Schmidt-Cassegrain telescope and photometer by M04. We solved the light curves individually using EBOP $\ddagger$ (Nelson \& Davis 1972; Popper \& Etzel 1981).

$\dagger$ Spectroscopic Binary Orbit Program written by Dr. Paul B. Etzel (http://mintaka.sdsu.edu/faculty/etzel/).

$\ddagger$ Eclipsing Binary Orbit Program written by Dr. Paul B. Etzel (http://mintaka.sdsu.edu/faculty/etzel/). 


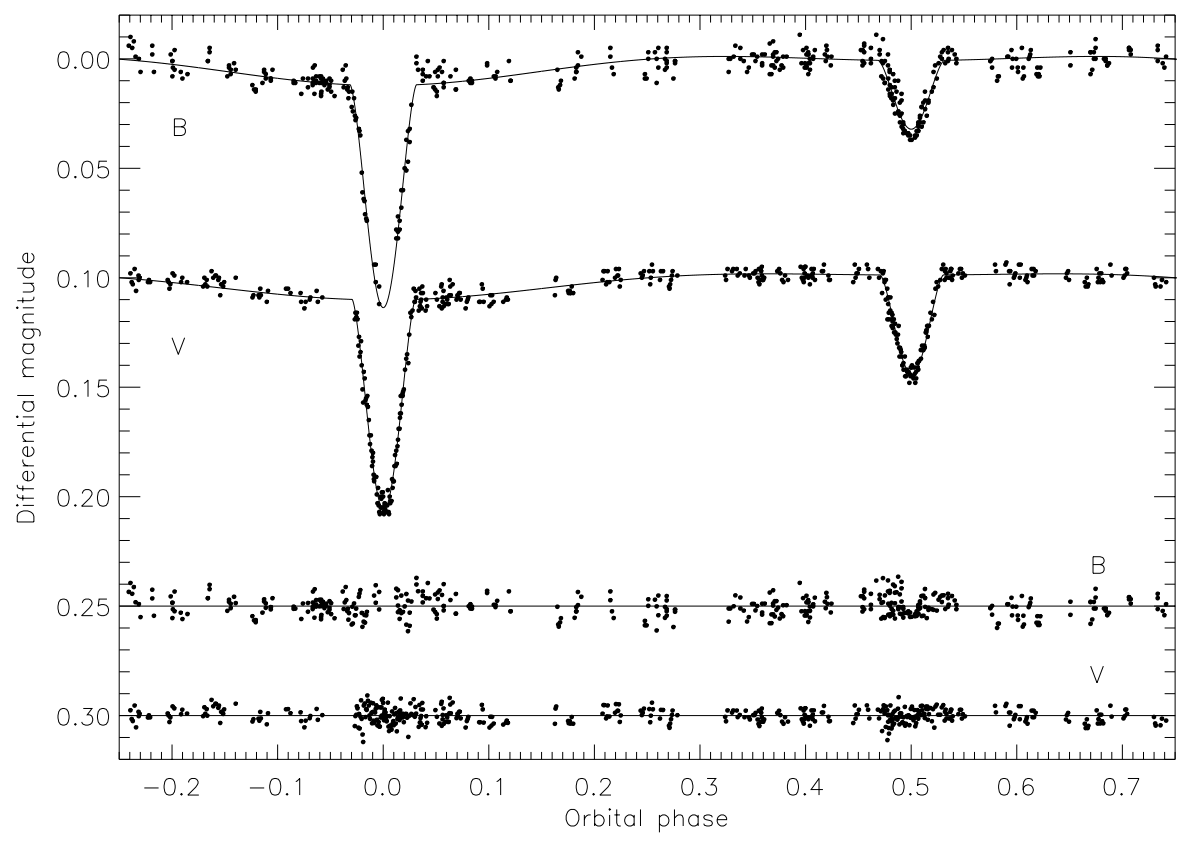

Figure 2. The M04 $B$ and $V$ light curves with our best fitted light curves overplotted. The $V$ light curve is shifted by $+0.1 \mathrm{mag}$ for clarity. The residuals of the fit are offset by $+0.25 \mathrm{mag}$ and +0.30 mag for the $B$ and $V$ light curves respectively. Note the poor fit around the secondary eclipse in the $B$ light curve due to scattered data.

This is a simple and efficient light curve fitting code in which the discs of the stars are modelled using biaxial ellipsoids. Filter-specific linear limb darkening coefficients were taken from van Hamme (1993), gravity darkening exponents, $\beta_{1}$, were fixed at 1.0 (Claret 1998) and the mass ratio was fixed at the spectroscopic value. The ephemeris given in M04 was used and the orbit was assumed to be circular. As there are no spectral lines due to a third star visible in the high-quality spectra of HD 23642, and third light, $L_{3}$, was negligible in initial photometric solutions, we have assumed $L_{3}=0$. However, as this quantity is not very well constrained by the light curves, we have also assessed the effect of assuming $L_{3}=0.05$ (in units of the total brightness of the eclipsing system) on the final result.

Initial light curve solutions using EBOP did not provide a good fit to the observations outside eclipse, so the size of the reflection effect on the secondary star was allowed to vary independently rather than being calculated from the system geometry. The light curves were also solved with the Wilson-Devinney code, using the 1998 version (WD98) in mode 2 and with a detailed treatment of the reflection effect. The $B$ and $V$ light curves were solved independently as before, except for the use of a logarithmic limb darkening law and fixing the stellar albedos at 1.0. The parameters derived from the light curve fits using EBOP and WD98 were very similar except for the ratio of the radii, which is poorly constrained by the light curves (see Fig. 3).

Considering the good agreement between the results of the EBOP and WD98 preliminary light curve fits, we used EBOP for further analysis. This code has two important advantages; firstly, a detailed error analysis is not prohibitively expensive in terms of computer time. Secondly, the philosophy of the EBOP code is to solve for the set of parameters most directly related to the light curve shape, leaving further analysis to the 

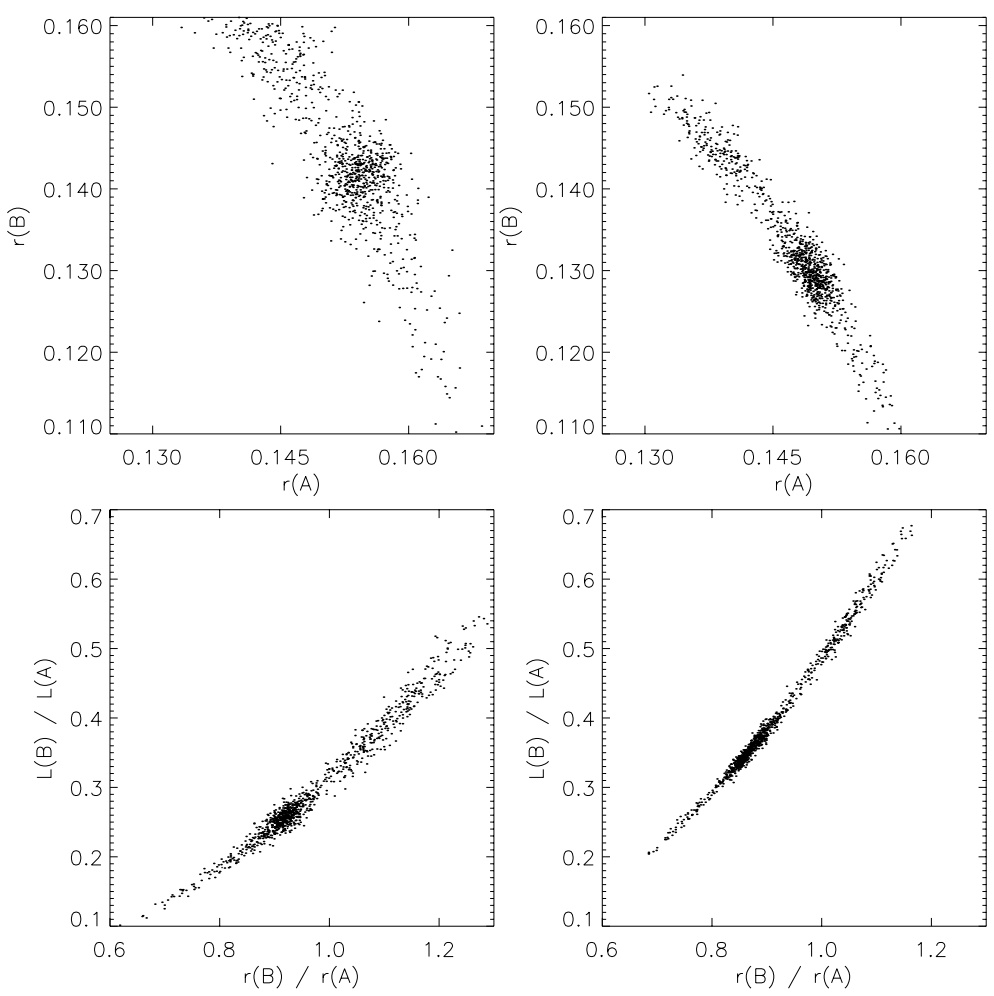

Figure 3. Results of the Monte Carlo analysis for the $B$ (left) and $V$ (right) light curves. The upper panels show primary radius versus secondary radius whilst the lower panels show the ratio of the radii versus the light ratios. All panels show that their parameters are strongly correlated, which is why our adopted solution uses a spectroscopic light ratio to break the degeneracy by allowing the ratio of the radii to be fixed during solution. Note the greater scatter of the synthetic solutions for the $B$ light curve, which is due to the greater observational errors. Only 1000 of the 10000 points have been plotted in each panel.

researcher. This allows a light curve solution for fixed values of the ratio of the radii and does not require the estimation of an effective temperature before solution.

The $B$ and $V$ light curve fits gave somewhat different results for the parameters in common - for example the sum of the radii, usually very well determined, differs by $5 \%$ between the two light curves. Attempts to solve this discrepancy, by manually rejecting discrepant data in the $B$ light curve, were unsuccessful so all photometric data have been retained. This difficulty was not mentioned by M04, who solved the $B$ and $V$ light curves simultaneously using the WD98 code.

Torres (2003) gave a preliminary monochromatic light ratio at $5187 \AA$ of $\frac{l_{\mathrm{B}}}{l_{\mathrm{A}}}=0.31 \pm$ 0.03. The final solution has been obtained by using this light ratio, converted to a $V$ filter light ratio using synthetic spectra from ATLAS9 model atmospheres, the effective temperatures found in Section 2, the surface gravities of M04, and a $V$ filter transmission function $\dagger$. The uncertainty of the resulting light ratio, $0.335 \pm 0.035$, includes possible systematic errors from the use of ATLAS9 model atmospheres. Note that the use of ATLAS9 model atmospheres rather than black bodies has a negligible effect on the resulting light ratio. This light ratio was fixed in the EBOP analysis of the $V$ light curve and used to

$\dagger$ The $V$ filter transmission function was taken from the Isaac Newton Group website http://www.ing.iac.es/Astronomy/astronomy.html. 
Table 4. Best-fitting parameters for the $V$ light curve found using EBOP and the light ratio of Torres (2003). The individual errors are from Monte Carlo simulations, with a fixed ratio of the radii, around the best-fitting light curves. The last line gives the adopted parameters.

\begin{tabular}{cccccc}
\hline $\begin{array}{l}\text { Light } \\
\text { ratio }\end{array}$ & $\begin{array}{c}\text { Ratio of the } \\
\text { radii, } k\end{array}$ & $\begin{array}{c}\text { Surface bright- } \\
\text { ness ratio, } J\end{array}$ & $\begin{array}{c}\text { Primary stellar } \\
\text { radius, } r_{1}(a)\end{array}$ & $\begin{array}{c}\text { Secondary stellar } \\
\text { radius, } r_{2}(a)\end{array}$ & $\begin{array}{c}\text { Orbital } \\
\text { inclination }\left({ }^{\circ}\right)\end{array}$ \\
\hline 0.300 & 0.8075 & $0.483 \pm 0.014$ & $0.1531 \pm 0.0009$ & $0.1237 \pm 0.0007$ & $78.04 \pm 0.08$ \\
0.335 & 0.8477 & $0.489 \pm 0.015$ & $0.1508 \pm 0.0009$ & $0.1278 \pm 0.0008$ & $77.90 \pm 0.08$ \\
0.370 & 0.8858 & $0.493 \pm 0.015$ & $0.1485 \pm 0.0009$ & $0.1315 \pm 0.0008$ & $77.79 \pm 0.08$ \\
0.335 & 0.8477 & $0.489 \pm 0.021$ & $0.1508 \pm 0.0032$ & $0.1278 \pm 0.0047$ & $77.90 \pm 0.19$ \\
\hline
\end{tabular}

Table 5. Best-fitting parameters for the $B$ light curve found using EBOP and the light ratio of Torres (2003). The individual errors are from Monte Carlo simulations, with a fixed ratio of the radii, around the best-fitting light curves. The last line gives the adopted parameters.

\begin{tabular}{cccccc}
\hline \multirow{2}{*}{$\begin{array}{c}\text { Ratio of the } \\
\text { radii, } k\end{array}$} & $\begin{array}{c}\text { Light } \\
\text { ratio }\end{array}$ & $\begin{array}{c}\text { Surface bright- } \\
\text { ness ratio, } J\end{array}$ & $\begin{array}{c}\text { Primary stellar } \\
\text { radius, } r_{1}(a)\end{array}$ & $\begin{array}{c}\text { Secondary stellar } \\
\text { radius, } r_{2}(a)\end{array}$ & Orbital \\
inclination $\left({ }^{\circ}\right)$
\end{tabular}

determine the ratio of the radii. This ratio of the radii was then used to solve the $B$ light curve. The results are given in Table 4 and Table 5 .

The uncertainties in the fitted parameters were estimated using a Monte Carlo method implemented for the study of the high-mass eclipsing binary V453 Cygni (Southworth et al. 2004b). This algorithm evaluates the correlations between different light curve parameters around the point of best fit, given the actual phases of observation and observational error. The resulting uncertainties are given in Table 4 and Table 5. The final values of the adjusted photometric parameters are $r_{1}=0.1538 \pm 0.0024, r_{2}=$ $0.1300 \pm 0.0037$ and $i=77^{\circ} .78 \pm 0.17$, where the quantities are the weighted means of the individual determinations from the $B$ and $V$ light curves. The best fit is plotted in Fig. 2.

Fig. 3 shows the results of the Monte Carlo analysis for the $B$ and $V$ light curves using the EBOP code and solving for the parameters including the ratio of the radii. The large parameter correlations illustrate the nature of the light curve and show why the spectroscopic light ratio of Torres (2003) was needed to allow us to fix the ratio of the radii for the final photometric solution. If the light curves are solved without using the light ratio of Torres (2003), the resulting fractional radii are $r_{1}=0.151 \pm 0.005$ and $r_{2}=0.135 \pm 0.007$; they are determined to accuracies no better than $3 \%$ and $5 \%$ respectively. The $\chi^{2}$ value of the light curve fits is approximately constant for values of the ratio of the radii between 0.8 and 1.0. The current light curves are not definitive by the criteria of Popper (2000). Our results agree with the results of M04 although our uncertainties are significantly larger than the formal errors quoted by these authors. This is despite the fact that we have incorporated extra information, a spectroscopic light ratio, into our analysis. Formal errors are known to be optimistic (e.g., Popper 1984). 
Table 6. Absolute dimensions of the components of HD 23642. The equatorial rotational velocities have been derived assuming that the values of M04 are projected rotational velocities and the orbital inclination derived in Section 3.

\begin{tabular}{lcl}
\hline & Primary star & Secondary star \\
\hline Mass $\left(\mathrm{M}_{\odot}\right)$ & $2.193 \pm 0.017$ & $1.550 \pm 0.014$ \\
Radius $\left(\mathrm{R}_{\odot}\right)$ & $1.831 \pm 0.030$ & $1.548 \pm 0.045$ \\
Surface gravity $\log g\left(\mathrm{~cm} \mathrm{~s}^{-1}\right)$ & $4.254 \pm 0.018$ & $4.249 \pm 0.029$ \\
Effective temperature $(\mathrm{K})$ & $9750 \pm 250$ & $7600 \pm 400$ \\
Equatorial rotational velocity $\left(\mathrm{km} \mathrm{s}^{-1}\right)$ & $38 \pm 1$ & $32 \pm 2$ \\
Synchronous velocity $\left(\mathrm{km} \mathrm{s}^{-1}\right)$ & $27.7 \pm 0.6$ & $31.8 \pm 0.9$ \\
$B$ & $7.109 \pm 0.044$ & $8.782 \pm 0.150$ \\
$V$ & $7.133 \pm 0.043$ & $8.320 \pm 0.100$ \\
\hline
\end{tabular}

\section{Absolute dimensions and comparison with stellar models}

Consideration of the final results from the photometric analysis (Table 4 and Table 5) and the results of the spectroscopic orbital analysis in Section 2 give the absolute dimensions of the two stars (Table 6). Our results are in reasonable agreement with the results of M04, and although the radius of the secondary star is somewhat larger, the two values are consistent within their uncertainties.

In Fig. 4 the observed properties of HD 23642 have been compared with predictions of the Granada stellar evolutionary models for $Z=0.01,0.02$ and 0.03 (Claret 1995, 1997; Claret \& Giménez 1995), adopting an age of $125 \mathrm{Myr}$ for the Pleiades (Stauffer, Schultz \& Kirkpatrick 1998). The positions of the components of HD 23642 in the mass-radius plane are consistent with the metal abundance of the Pleiades being solar or slightly supersolar. This confirms the atmospheric solar iron abundance determined by Boesgaard \& Friel (1990) from high-resolution spectroscopy of F dwarfs, but is in disagreement with suggestions that the 'short' and 'long' Pleiades distances could be reconciled by adopting a low metal abundance for the cluster.

The components of HD 23642 cannot be fitted in the mass-radius plane by models of metal abundance or helium abundance significantly different from the solar abundances; for a solar chemical composition the predictions of the Granada stellar evolutionary models are consistent with the dimensions of HD 23642 for ages between 125 and 175 Myr. Predictions of the Padova (Bressan et al. 1993) and Cambridge (Pols et al. 1998) stellar models are in agreement with this conclusion, but the Geneva (Schaller et al. 1992) evolutionary models predict a smaller radius for the secondary star than observed.

\section{The distance to the Pleiades}

\subsection{Distance using bolometric corrections}

The traditional method of determining the distance of an eclipsing binary is to calculate the luminosity of each star using the usual formula

$$
L=4 \pi \sigma R^{2} T_{\mathrm{eff}}^{4}
$$

where $R$ is the stellar radius and $\sigma$ is the Stefan-Boltzmann constant. Then the absolute bolometric magnitude for each star is found using the definition

$$
M_{\mathrm{bol}}=-2.5 \log \frac{L}{L_{\odot}}+M_{\mathrm{bol} \odot}
$$




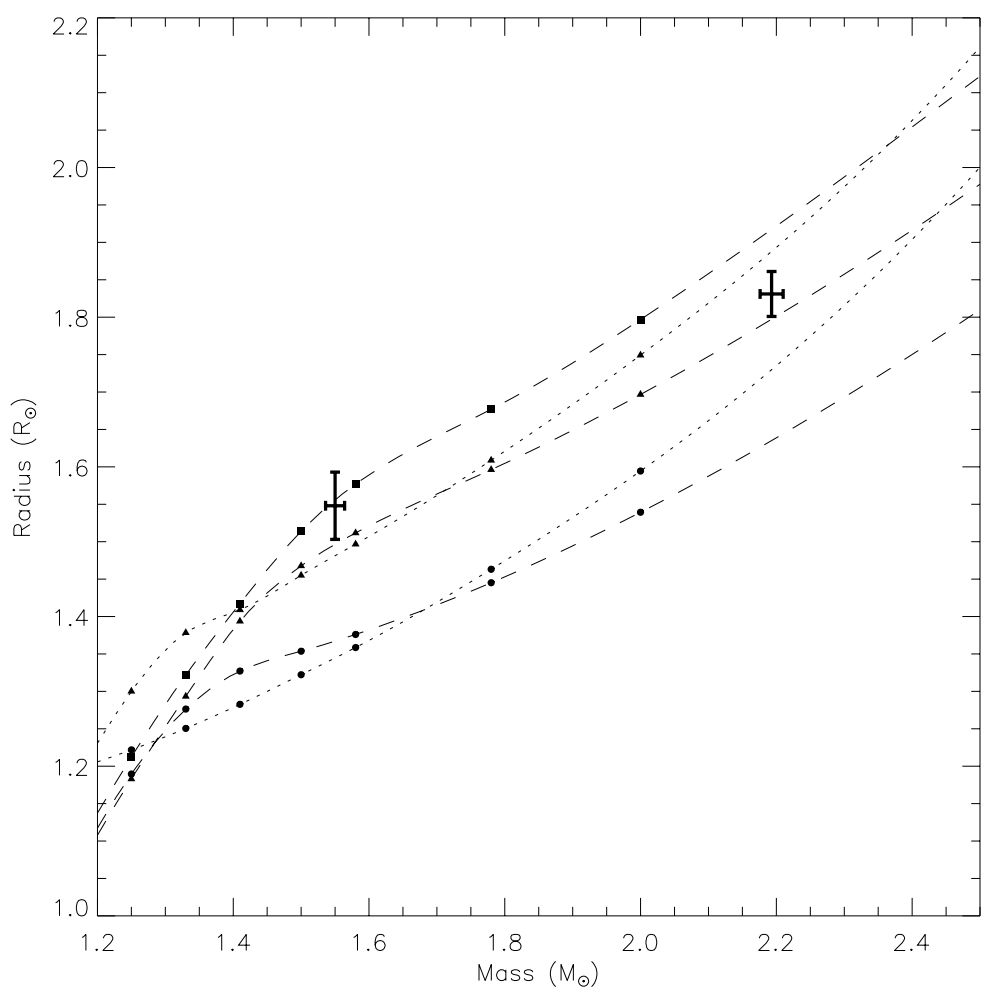

Figure 4. Comparison between the observed properties of HD 23642 and the Granada models for metal abundances of $Z=0.01$ (circles), $Z=0.02$ (triangles) and $Z=0.03$ (squares). Predictions for normal helium abundances are plotted with dashed lines and helium-rich model predictions ( $Y=0.34$ and 0.38 for $Z=0.01$ and 0.02 respectively) are plotted using dotted lines. An age of $125 \mathrm{Myr}$ was assumed.

The absolute visual magnitude, $M_{V}$, of each star is calculated and the distance, $d$ (parsecs), derived using

$$
V_{0}-M_{V}=\left(V-A_{V}\right)-\left(M_{\mathrm{bol}}-B C\right)=5 \log d-5
$$

where $V$ is the apparent visual magnitude of the star, $A_{V}$ is the total visual extinction, $V_{0}$ is the dereddened apparent visual magnitude, and $B C$ is the bolometric correction. This provides a distance to each star or, alternatively, the absolute visual fluxes of the two stars can be summed to find the total absolute visual magnitude of the system.

There are several problems with deriving distances by using bolometric corrections:

(a) The effective temperature scale must be fundamental to avoid systematic errors.

(b) $M_{\text {bol } \odot}$ and $L_{\odot}$ are not defined to an arbitrary precision.

(c) Bolometric corrections are calculated using stellar models so the final result has a model dependence.

(d) The final distance uncertainty can be very sensitive to the uncertainties in the effective temperatures and must also incorporate uncertainties in the bolometric corrections used.

(e) Consistent values of $M_{\mathrm{bol} \odot}$ and $L_{\odot}$ must be adopted to avoid systematic errors caused by the use of different zeropoints.

Adopting the astrophysical parameters of the system from Tables 1 and 6 , bolometric corrections from Bessell, Castelli \& Plez (1998), and uncertainties in bolometric corrections 
from consideration of other tabulations in the literature, we find a distance to HD 23642 of $138.1 \pm 4.7 \mathrm{pc}$. For the primary component of HD 23642 , the uncertainty due to its effective temperature is actually reduced due to the form of the bolometric correction function around $10000 \mathrm{~K}$. The main contribution to the overall uncertainty ( $4.5 \mathrm{pc}$; note that the individual uncertainties are added in quadrature) comes from the uncertainty in the effective temperature of the secondary star. Whilst this is an acceptable result to adopt for the final distance to HD 23642, it is still affected by (probably small) systematic error due to the use of bolometric corrections calculated from theoretical models.

M04 found the distance to HD 23642, from the above method and the absolute bolometric magnitudes of the stars given by the Wilson-Devinney code, to be $131.9 \pm 2.1 \mathrm{pc}$. The bolometric magnitudes given by M04 are consistent with the values $M_{\text {bol } \odot}=4.77$ and $L_{\odot}=3.906 \times 10^{26} \mathrm{~W}$, which are adopted in WD98. This $M_{\text {bol } \odot}$ value is different to the standard value of 4.75 (Hilditch 2001) and is also different to the value of 4.74 adopted by Bessell et al. (1998), from which M04 have taken their bolometric corrections. If we adopt the temperatures and radii of $\mathrm{M} 04$, and the $M_{\mathrm{bol} \odot}=4.74$ and bolometric corrections given by Bessell et al., we find a distance of $134.5 \pm 2.5$ pc. However, adoption of the bolometric corrections of Flower (1996), and $M_{\mathrm{bol} \odot}=4.75$ (as Flower does not give the value he adopted), gives a distance of $135.4 \pm 2.6 \mathrm{pc}$, which is $0.9 \mathrm{pc}$ different to the previous value.

\subsection{Distance using surface brightness calibrations}

An alternative to considering the unobserved parts of the stellar spectral energy distributions is to use empirical relations between effective temperature and the visual surface brightness of a star. In this method the angular diameter of the star is compared to its linear diameter, determined from photometric analysis, to find the distance. The main source of uncertainty in this method is usually the light ratio, found from the photometric analysis, needed to calculate the individual magnitudes of the components of the dEB from the apparent magnitude of the system. If the effective temperatures of the two stars are known, this difficulty can be bypassed by comparing the apparent magnitude of the system to the flux-weighted combined angular diameter of the two stars.

The surface brightness of a star is defined to be

$$
S_{m_{\lambda}}=m_{\lambda}-5 \log \phi
$$

where $m_{\lambda}$ is the apparent magnitude in filter $\lambda$ and $\phi$ is the angular diameter of the star in milli-arcseconds. The zeroth-magnitude angular diameter is defined to be

$$
\phi^{\left(m_{\lambda}=0\right)}=\phi \times 10^{\frac{m_{\lambda}}{5}}=\frac{S_{m_{\lambda}}}{5}
$$

(van Belle 1999), i.e., the angular diameter a star would have if its apparent magnitude in the filter $m_{\lambda}$ were zero. From consideration of the definitions of surface brightness and angular diameter, it can be shown that the distance to a dEB is given by

$$
d=10^{0.2 m_{\lambda}} \sqrt{\left[\frac{2 R_{\mathrm{A}}}{\phi_{\mathrm{A}}^{\left(m_{\lambda}=0\right)}}\right]^{2}+\left[\frac{2 R_{\mathrm{B}}}{\phi_{\mathrm{B}}^{\left(m_{\lambda}=0\right)}}\right]^{2}}
$$

where, for a distance given in parsecs, the stellar radii $R_{\mathrm{A}}$ and $R_{\mathrm{B}}$ are given in $\mathrm{AU}$, the zeroth-magnitude angular diameters $\phi_{\mathrm{A}}^{\left(m_{\lambda}=0\right)}$ and $\phi_{\mathrm{B}}^{\left(m_{\lambda}=0\right)}$ are given in arcseconds and $\lambda$ represents a broad-band filter passband. Calibrations for $\phi^{\left(m_{\lambda}=0\right)}$ are given in terms of effective temperature by Kervella et al. (2004, hereafter KTDS04) (where they are denoted using $\mathrm{ZMLD}_{\lambda}$ ) for the broad-band $U B V R I J H K L$ filters. The calibrations with 
Table 7. The results and individual error budgets for distance estimates using the effective temperatures and overall apparent magnitudes of the HD 23642 system in the $B V J H K$ filters. All distances are given in parsecs and the total uncertainties are the sums of the individual uncertainties added in quadrature. The observational scatter around the calibrations is denoted as 'cosmic' scatter.

\begin{tabular}{lrrrrr}
\hline Source of uncertainty & $B$ & $V$ & $J$ & $H$ & $K$ \\
\hline Effective temperature of the primary star & 4.8 & 3.3 & 1.7 & 0.7 & 0.7 \\
Effective temperature of the secondary star & 3.8 & 3.5 & 2.1 & 1.5 & 1.4 \\
Apparent magnitude of the system & 2.1 & 1.8 & 2.2 & 2.1 & 2.1 \\
Radius of the primary star & 1.9 & 1.7 & 1.5 & 1.4 & 1.4 \\
Radius of the secondary star & 0.9 & 1.0 & 1.3 & 1.5 & 1.5 \\
Intrinsic "cosmic" scatter in the calibration & 9.0 & 8.3 & 1.7 & 1.9 & 1.4 \\
Total uncertainty in distance & 11.2 & 10.0 & 4.4 & 3.9 & 3.6 \\
Distance & 142.8 & 141.4 & 139.6 & 138.4 & 139.1 \\
\hline
\end{tabular}

the least scatter involve the $J H K L$ filters. For example, the calibrations for the $V$ and $K$ filters are

$$
\begin{array}{ll}
\log \phi^{(V=0)}=3.0415\left(\log T_{\text {eff }}\right)^{2}-30.9671\left(\log T_{\text {eff }}\right)+53.7010 & \sigma=5.9 \% \\
\log \phi^{(K=0)}=0.8470\left(\log T_{\text {eff }}\right)^{2}-7.0790\left(\log T_{\text {eff }}\right)+15.2731 & \sigma<1.0 \%
\end{array}
$$

For the $K$ filter calibration the scatter is undetectable at a level of $1 \%$ so we conservatively adopt a fitting uncertainty of $1.0 \%$. We have applied the calibrations for the $B$ and $V$ filters (using Tycho apparent magnitudes transformed to the Johnson system) and for the $J H K$ filters (using 2MASS data transformed to the SAAO system). As there is no "standard" infrared photometric system, the calibrations of KTDS04 use data from several different $J H K L$ systems, so the systematic uncertainty of not having a standard system is already included in the quoted scatter in the calibrations. For the A stars only, the scatter around the $B$ and $V$ calibrations is much smaller than the overall scatter quoted, so for HD 23642 the $B$ and $V$ distance uncertainties are overestimated by a factor of about two.

The distances found using equation 5.6 and the KTDS04 calibrations are given in Table 7. Note that the uncertainties in the $J H K$ calibration distances are much smaller than in the $B V$ calibration distances. We will adopt the $K$ filter calibration distance of $139.1 \pm 3.6 \mathrm{pc}$ as our final distance to HD 23642 and so to the Pleiades. Note that we cannot treat any of the distance estimates investigated above as being independent of each other as they all incorporate an effective temperature of one or both of the stars in HD 23642 .

One shortcoming of finding distances using equation 5.6 is that the effective temperature scales used in analysis of the $\mathrm{dEB}$ and for the calibration must be the same to avoid systematic errors. This is, however, a more relaxed constraint on the effective temperature scale than that involved in finding distance through absolute bolometric magnitude. We note that our effective temperature uncertainties include contributions due to possible spectral peculiarity and systematic offset relative to the (inhomogeneous) effective temperatures used in the KTDS04 calibration. The uncertainty in distance could be reduced by further observations and estimations of the effective temperatures of the two stars using the same technique as for the stars used to calibrate the surface brightness relations.

A way of avoiding systematic errors in the determination of effective temperatures is to use calibrations between surface brightness and colour indices for the individual stars. 
This method is of only minor interest here but gives a distance of $138 \pm 19 \mathrm{pc}$. The large uncertainty in this value is because we only have $B-V$, which is a poor surface brightness indicator due to the small wavelength difference between the $B$ and $V$ filters and because the $B$ filter is sensitive to metallicity due to the effects of line blanketing. Good surface brightness indicators are the $B-L$ and $V-K$ indices (KTDS04; Di Benedetto 1998), where the observed scatter becomes much less than $1 \%$, and the Strömgren $c_{1}$ index (Salaris \& Groenewegen 2002). This method would also require additional photometry in several filters, including $B$ or $V$, as well as a significant number of observations - or a complete light curve - in the $K$ or $L$ filters.

\section{Conclusion}

The distance of the Pleiades open cluster is currently controversial. The 'long' distance of the Pleiades is $132 \pm 3 \mathrm{pc}$ and is supported by main sequence fitting, the distance of the astrometric binary Atlas, and by ground-based trigonometrical parallax measurements. The 'short' distance is $120 \pm 3 \mathrm{pc}$ and comes from trigonometrical parallaxes observed by the Hipparcos space satellite. It has been suggested that the two distances could be reconciled if the Pleiades cluster is metal-poor, but determinations of the metal abundances of Pleiades F dwarfs suggest that the cluster has a solar iron abundance.

We have reanalysed the observational data on HD 23642 to determine absolute dimensions and effective temperatures. Our uncertainties mainly derive from the adoption of a spectroscopic light ratio to determine an otherwise poorly known ratio of the radii, but Monte Carlo simulations have also been undertaken to estimate the remaining uncertainties in the light curve fits. As the spectroscopic light ratio is preliminary, our results should also be viewed as such.

By comparing the radii of the components of HD 23642 to theoretical evolutionary models we find that the system has a solar or slightly supersolar metal abundance and a normal helium abundance, which removes the possibility that the 'long' and 'short' distance scales could be reconciled by adopting a low metal abundance or high helium abundance for the Pleiades cluster. This result is almost independent of the spectroscopic light ratio as the important quantity, the sum of the radii, is relatively well determined by the light curves.

From the use of bolometric corrections and our own temperatures and radii, we have calculated the distance to HD 23642 to be $138.1 \pm 4.7 \mathrm{pc}$. The error given is a random error, but there is also a small systematic error due to the use of model atmospheres to calculate bolometric corrections and effective temperatures. M04 found a distance of $131.9 \pm 2.1 \mathrm{pc}$ using their own astrophysical data. Depending on the bolometric corrections adopted, we find that their temperatures and radii imply a distance to the dEB of $134.5 \pm 2.5 \mathrm{pc}$ or $135.4 \pm 2.6 \mathrm{pc}$. This difference is probably due to different assumed values for the solar absolute bolometric magnitude and effective temperature.

We have found the distance to HD 23642 by using the effective temperature - surface brightness calibration of KTDS04. This method avoids the systematic errors due to the use of bolometric corrections and the calculation of luminosities. We find a distance of $139.1 \pm 3.6 \mathrm{pc}$, where the biggest contributor to the uncertainty comes from the apparent magnitude measurement. There may be a slight systematic error in this result due to our method of determining the effective temperatures of HD 23642, but our uncertainties include this and the systematic error is certainly smaller than in the determination of distances by the use of bolometric corrections. An alternative distance of $138 \pm 19 \mathrm{pc}$ has been found by the use of the surface brightness $-(B-V)$ calibration of Di Benedetto (1998). This result is entirely empirical but its precision is low. The $B$ filter magnitude 
is also dependent on the photospheric metal abundance so could be affected by the metallic-lined nature of the secondary star.

Our distance to HD 23642 is slightly larger than the 'long' distance to the Pleiades but is in full agreement with the constraints on the Pleiades distance from study of the astrometric binary Atlas (Pan et al. 2004). The distance derived by M04 is also in agreement with the 'long' distance scale. Our distance, and that of M04, is inconsistent with the Hipparcos 'short' distance, and indeed with the Hipparcos parallax distance of the dEB. We also confirm that the Pleiades has a solar metal abundance, removing the possibility of composition effects being used to reconcile the two distances.

Further observations of HD 23642, to determine accurate dimensions, would provide very precise metal abundance and effective temperature measurements for the component stars. This would reduce the uncertainty in its distance and allow further investigation of the system, which is itself an interesting object due to the metallic-lined nature of the secondary star. Further infrared or Strömgren observations, however, would allow the use of entirely empirical surface brightness relations for the calculation of the distance to HD 23642.

Distance estimation using the surface brightness calibrations of KTDS04 appears to have a lot of potential. Distances accurate to $2 \%$ could be obtained from definitive analysis of dEBs incorporating infrared $J H K$ photometry. The study of dEBs in open clusters is also a powerful tool for inferring the chemical composition of the clusters.

\section{Acknowledgements}

The authors would like to thank Dr. U. Munari for making his data on HD 23642 freely available, and for helpful discussions. This publication makes use of data products from the Two Micron All Sky Survey, which is a joint project of the University of Massachusetts and the Infrared Processing and Analysis Center/California Institute of Technology, funded by the National Aeronautics and Space Administration and the National Science Foundation. JS acknowledges financial support from PPARC in the form of a postgraduate studentship. The authors acknowledge the data analysis facilities provided by the Starlink Project which is run by CCLRC on behalf of PPARC. The following internet-based resources were used in research for this paper: the NASA Astrophysics Data System; the SIMBAD database operated at CDS, Strasbourg, France; the VizieR service operated at CDS, Strasbourg, France; and the ar $\chi$ iv scientific paper preprint service operated by Cornell University.

\section{References}

Abt, H. A. 1958 ApJ 128, 139-141.

Abt, H. A. \& Levato, H. 1978 PASP 90, 201-203.

Andersen, J. 1991 A $\& A$ Review 3, 91-126.

Argelander, F. 1903 Bonner Durchmusterung des nordlichen Himmels, Eds Marcus and Weber's Verlag, Bonn.

Bessell, M. S. 2000 PASP 112, 961-965.

Bessell, M. S., Castelli, F. \& Plez, B. 1998 A\&A 333, 231-250.

Boesgaard, A. M. \& Friel, E. D. 1990 ApJ 351, 467-479.

Bressan, A., Fagotto, F., Bertelli, G. \& Chiosi, C. 1993 A\& AS 100, 647-664.

Cannon, A. J. \& Pickering, E. C. 1918 Annals of Harvard College Obs. 91, 1-290.

Castellani, V., Degl'Innocenti, S., Prada Moroni, P. G. \& Tordiglione, V. 2002 MNRAS 334, $193-197$.

Claret, A. 1995 A\&AS 109, 441-446.

Claret, A. 1997 A $B A S$ 125, 439-443. 
Claret, A. 1998 A\&AS 131, 395-400.

Claret, A. \& Giménez, A, 1995 A $\mathscr{J} A S$ 114, 549-556.

Crawford, D. L. \& Perry, C. L. 1976 AJ 81, 419-426.

Di Benedetto, G. P. 1998 A\&A 339, 858-871.

Flower, P. J. 1996 ApJ 469, 355-365.

Gatewood, G., de Jonge, J. K. \& Han, I. 2000 ApJ 533, 938-943.

Grenon, M. 1999 in Garcia Lopez R. J., Rebolo R., Zapaterio Osorio M. R., eds, ASP Conf. Ser. Vol. 223, Proc. 11th Cambridge Workshop on Cool Stars. Astron. Soc. Pac., San Francisco, p. 359.

Griffin, R. F. 1995 Journal Royal Ast. So.c. Canada 89, 53-62.

Hertzsprung, E. 1947 Ann. Sterrew. Leiden 19, 3.

Hilditch, R. W. 2001 An Introduction to Close Binary Stars Cambridge University Press, p. 281.

Johnson, H. L. 1957 ApJ 126, 121-133.

Kervella, P., Thévenin, F., Di Folco, E. \& Ségransan, D. 2004 A $\& A$ 426, 297-307.

Makarov, V. V. 2002 AJ 124, 3299-3304.

Meynet, G., Mermilliod, J.-C. \& Maeder, A. 1993 A\& $A S$ 98, 477-504.

Moro, D. \& Munari, U. $2000 A \& A S$ 147, 361-628.

Munari, U., Dallaporta, S., Siviero, A., Soubiran, C., Fiorucci, M. \& Girard, P. 2004 A\&A 418, L31-L34 (M04).

Nelson, B. \& Davis, W. D. 1972 ApJ 174, 617-628.

O'Dell, M. A., Hendry, M. A. \& Collier Cameron, A. 1994 MNRAS 268, 181-193.

Pan, X., Shao, M. \& Kulkarni, S. S. 2004 Nature 427, 326-328.

Pearce, J. A. 1957 Publ. Dom. Astr Obs. 10, 435-445.

Perryman, M. A. C., et al. 1997 A\&SA 323, L49-L52.

Pinsonneault, M. H., Stauffer, J., Soderblom, D. R., King, J. R. \& Hanson, R. B. 1998 ApJ 504, 170-191.

Pols, O. R., Schröder, K.-P., Hurley, J. R., Tout, C. A. \& Eggleton, P. P. 1998 MNRAS 298, $525-536$.

Popper, D. M. $1984 A J$ 89, 132-144.

Popper, D. M. $2000 A J$ 119, 2391-2402.

Popper, D. M. \& Etzel, P. B. 1981 AJ 86, 102-120.

Robichon, N., Arenou, F., Mermilliod, J.-C. \& Turon, C. 1999 A\&A 345, 471-484.

Salaris, M. \& Groenewegen, M. A. T. 2002 A\& A 381, 440-445.

Schaller, G., Schaerer, D., Meynet, G. \& Maeder, A. 1992 AESAS 96, 269-331.

Soderblom, D. R., King, J. R., Hanson, R. B., Jones, B. F., Fischer, D., Stauffer, J. \& Pinsonneault, M. H. 1998 ApJ 504, 192-199.

Southworth, J., Maxted, P. F. L. \& Smalley, B. 2004a MNRAS 349, 547-559.

Southworth, J., Maxted, P. F. L. \& Smalley, B. 2004b MNRAS 351, 1277-1289.

Stauffer, J. R., Schultz, G. \& Kirkpatrick J. D. 1998 ApJ 499, L199-L203.

Stello, D. \& Nissen, P. E. 2001 A $\& A$ 374, 105-115.

Torres, G. 2003 IBVS $\mathbf{5 4 0 2}$.

Vandenberg, D. A. \& Bridges, T. J. 1984 ApJ 278, 679-688.

van Belle, G. T. 1999 PASP 111, 1515-1523.

van Hamme, W., 1993, AJ, 106, 2096. 1993 AJ 106, 2096-2117.

van Leeuwen, F. $1999 A \& A$ 341, L71-L74.

Wilson, R. E. \& Devinney, E. J. 1971 ApJ 166, 605-619.

Wilson, R. E. 1993 New Frontiers in Binary Star Research: Pacific Rim Colloquium. Astron. Soc. Pac., San Francisco, 91-126.

Zahn J.-P. 1977 A $G A$ 57, 383-394. 


\section{Discussion}

FRITz BenEDICT: First, I'm very pleased that we agree within our error bars! Secondly, a question: What's the observational evidence for a third light in the system?

John Southworth: None. We cannot assume it's not there. It might be; it's just that we can't see it.

FRITZ Benedict: So it's just the error bars, basically?

John SouthworTh: It makes a negligible difference, this assumption of $5 \%$. It only adds about a tenth to the uncertainties in the radii. But it's important to take into account, because there's no physical basis to assume that it's not there at all.

Don KuRTz: You think that the primary star is a silicon star. A silicon star in a binary that short is very unusual, but let's assume that it is true. If so, it's magnetic. If it's magnetic, it's got spots, and I notice you particularly have a problem fitting your secondary eclipse. A spot might handle that problem, so that's a little bit of extra you can throw in, at least at secondary eclipse time.

John Southworth: I do not agree with that entirely. Firstly, it may have a slightly enhanced silicon abundance. The spectral classification is quite old and we've not confirmed it so far, because we've only been at this data for about a month. Secondly, if I go back to the fitting of our secondary eclipse, the best fit does not appear to pass through the locus of the points. However, if you look at the residuals, you'll find it's not the problem with the model; I believe that's a problem with the photometry. I can improve the fit by rejecting data selectively. I'm not very keen on doing that. I should also point out that Munari et al. did not have this problem because they solved the data simultaneously, and the $V$ light curve is a lot better defined than the $B$ light curve, so it forced the results of the $B$ light curve to where you think the data should be. I'm not convinced that's a problem with the model. 


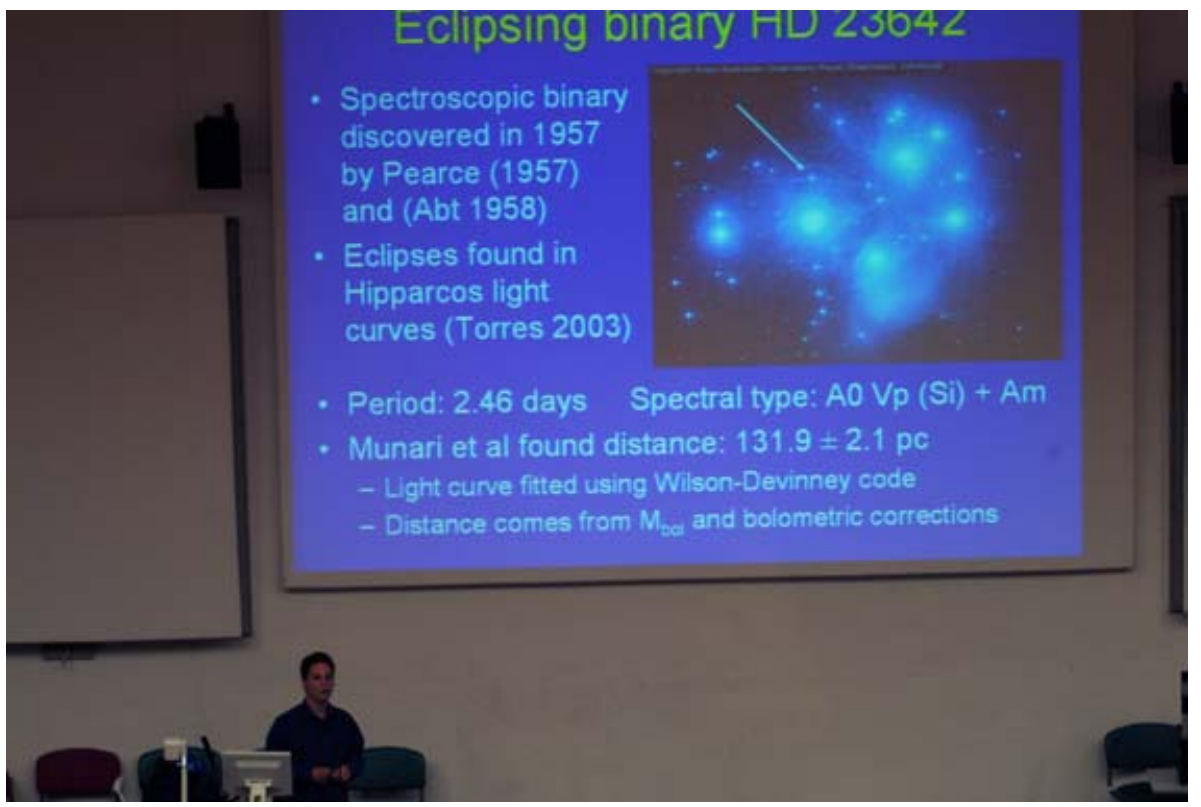

John Southworth presenting his paper

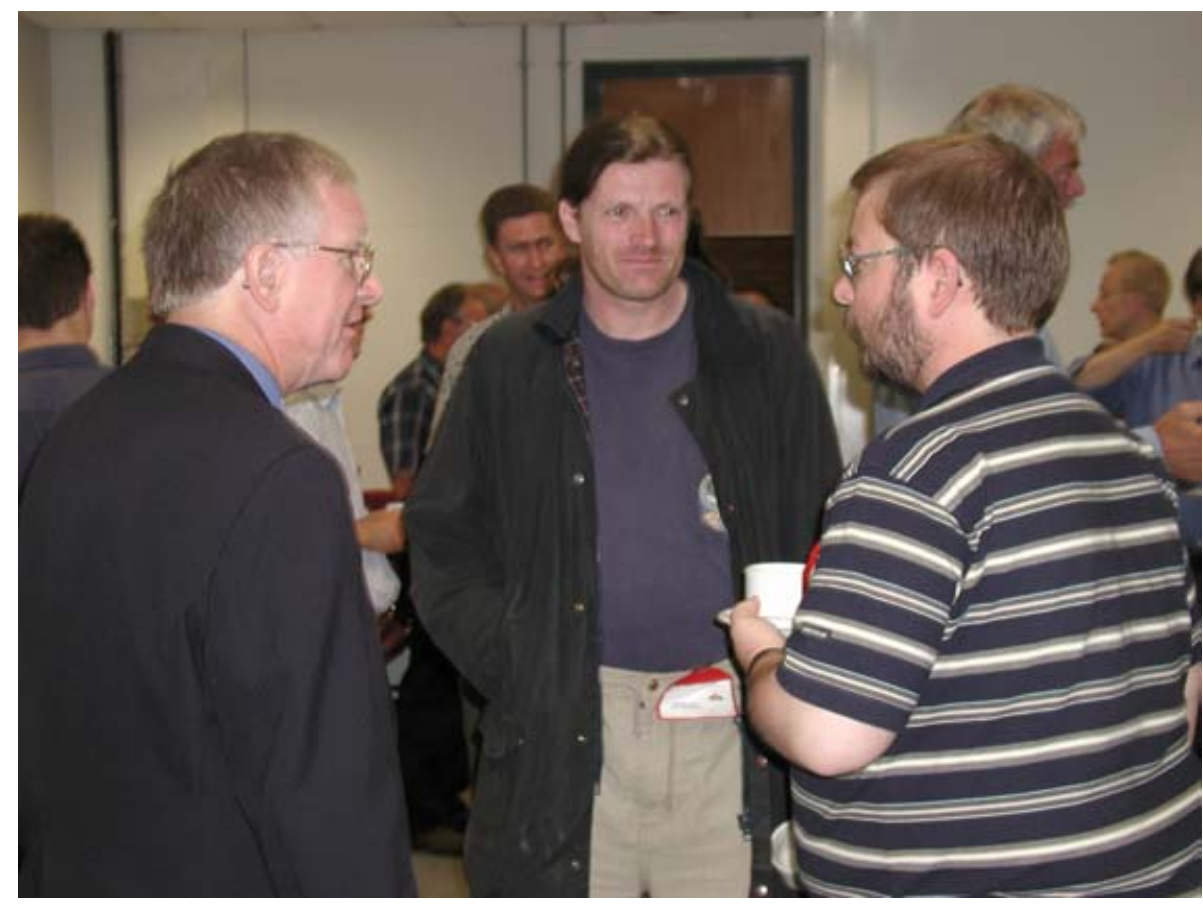

Fritz Benedict, Ricky Smart and Dimitri Pourbaix 